\title{
Texture Controls of Self-Constructive Tri-layered Composites
}

\author{
T. Nagase and O. Abe \\ The Research Center for Superplasticity, Faculty of Engineering, Ibaraki University, 4-12-1 Nakanarusawa, Hitachi \\ 316-8511, Japan \\ Fax: 81-0294-38-5087, e-mail: abe@mx.ibaraki.ac.jp
}

\begin{abstract}
Alumina-based composite containing $1 \mathrm{vol} \% \mathrm{NiAl}$ has been fabricated to study the toughening mechanism due to the oxidation-derived metamorphic layer on the surface. The development of the network structure substituting the boundary of the matrix $-\mathrm{Al}_{2} \mathrm{O}_{3}$ with the oxidation product $\mathrm{NiAl}_{2} \mathrm{O}_{4}$ induces enhanced crack deflection to improve the fracture toughness and strength from $3.6 \mathrm{MPa} \cdot \mathrm{m}^{1 / 2}$ and $539 \mathrm{MPa}$ (as-sintered) to $6.4 \mathrm{MPa} \cdot \mathrm{m}^{1 / 2}$ and $701 \mathrm{MPa}$ at the oxidation time of $5 \mathrm{~h}$. However, excess oxidation causes the diffusion of the surface oxidation products into the bulk. The disappearance of the network structure results in the decrease of the toughness and strength.
\end{abstract}

Key words: fracture toughness, compressive stress, $\mathrm{NiAl} / \mathrm{Al}_{2} \mathrm{O}_{3}$, tri-layered composite, network structure

\section{INTRODUCTION}

Improvement of the fracture toughness is important for the practical applications of engineering ceramics. Layered structure is one of the candidate textures for toughening the ceramic materials under two typical mechanisms [1], [2], (1) the enhanced crack deflection along the layer interfaces and (2) the surface compressive stress. However, the elastic mismatch for crack deflection sometimes causes the delaminations at the layer interfaces and the thermal expansion mismatch providing the compressive stress disappears at elevated temperatures. The authors have demonstrated another way generating compressive stress on the surface layer of $\mathrm{NiAl} / \mathrm{Al}_{2} \mathrm{O}_{3}$ composites by the volume expansion due to the following reactions.

$$
\begin{aligned}
& 2 \mathrm{NiAl}+5 / 2 \mathrm{O}_{2}=\mathrm{NiAl}_{2} \mathrm{O}_{4}+\mathrm{NiO} \\
& \mathrm{NiO}+\mathrm{Al}_{2} \mathrm{O}_{3} \text { (grain boundary) } \\
& =\mathrm{NiAl}_{2} \mathrm{O}_{4} \text { (boundary network) }
\end{aligned}
$$

Metamorphic layers consisting of $\mathrm{NiAl}_{2} \mathrm{O}_{4}$ boundary and $\mathrm{Al}_{2} \mathrm{O}_{3}$ grains have been formed on the surface of the oxidized NiAl $/ \mathrm{Al}_{2} \mathrm{O}_{3}$ materials. These tri-layer structured composites indicate the strictly joined interfaces due to the compositional gradient at the reaction front. The complete oxidation of $\mathrm{NiAl}$ on the surface of $10 \mathrm{vol} \% \mathrm{NiAl} / \mathrm{Al}_{2} \mathrm{O}_{3}$ material (NA10) provided the compressive stress of $128 \mathrm{MPa}$ in the metamorphic layer with the thickness of $1 \sim 3 \%$ of the specimens. The effective fracture toughness of this oxidized material was improved to $8 \mathrm{MPa} \cdot \mathrm{m}^{1 / 2}$ with the good agreement with the calculated values from the compressive stress. However, the $5 \mathrm{vol} \% \mathrm{NiAl} / \mathrm{Al}_{2} \mathrm{O}_{3}$ showed the inexpectedly higher fracture toughness of $8 \mathrm{MPa} \cdot \mathrm{m}^{1 / 2}$ than the estimation from the compressive stress. This difference suggested the additional contribution of the oxidation irrespective of the compressive stress in the metamorphic layers. In the present work, $\mathrm{Al}_{2} \mathrm{O}_{3}$-based composite containing a very small amount of $\mathrm{NiAl}(1 \mathrm{vol} \%)$ has been prepared to limit the compressive stress to negligible values, and the mechanical properties are discussed in relation to the development of the metamorphic layer.

\section{EXPERIMENTAL}

The 1vol.\% $\% \mathrm{NiAl} / \mathrm{Al}_{2} \mathrm{O}_{3}$ (NA01) powder was prepared by diluting a $10 \mathrm{vol} . \% \mathrm{NiAl} / \mathrm{Al}_{2} \mathrm{O}_{3}$ (NA10) powder synthesized via a chemical precipitation route with $\mathrm{Al}_{2} \mathrm{O}_{3}$ powder ( $1: 9$, in the volume ratio) by mechanical mixing. At first, the precursor for NA10 was synthesized by pouring a water/methanol solution $(1: 1)$ of $\mathrm{NiCl}_{2}$ and $\mathrm{AlCl}_{3}$ to the precipitant solution dispersing a fine $\alpha-\mathrm{Al}_{2} \mathrm{O}_{3}$ powder as the matrix at $70{ }^{\circ} \mathrm{C}$. The obtained precipitate mixture, $\left[\mathrm{Ni}\left(\mathrm{N}_{2} \mathrm{H}_{4}\right)_{2} \mathrm{Al}\right](\mathrm{OH})_{1.3}\left(\mathrm{C}_{6} \mathrm{H}_{5} \mathrm{COO}\right)_{3.7}$ $+\left[\alpha-\mathrm{Al}_{2} \mathrm{O}_{3}\right]_{\text {powder, }}$, was filtered, washed by the solvent and dried under the vacuum. NiAl was synthesized by the heat-treatment at $1360{ }^{\circ} \mathrm{C}$. in $\mathrm{Ar}$ following to the decomposition of organic groups at $600^{\circ} \mathrm{C}$. The residual carbon was removed by heating it at $580^{\circ} \mathrm{C}$ in air. The details of the preparation process of the powder were described elsewhere [3], [4]. The NA10 powder was mixed with $\mathrm{Al}_{2} \mathrm{O}_{3}$ powder mechanically. The NA01 powder was pre-formed to rectangular bars $(5 \times 5 \times$ $22 \mathrm{~mm}$ ), CIP-ed at $150 \mathrm{MPa}$, and sintered at $1430{ }^{\circ} \mathrm{C}$ for $4 \mathrm{~h}$ in Ar. The sintered materials were machined to test bars with the cross-sectional size of $3 \mathrm{~mm} \times 4 \mathrm{~mm}$, and finished by polishing with a $0.06 \mu \mathrm{m} \mathrm{SiO}_{2}$-suspension and chamfering. Tri-layer structure was introduced to the specimens by the oxidation at $1250{ }^{\circ} \mathrm{C}$ for $2.5 \mathrm{~h} \sim 20 \mathrm{~h}$ in air. The sintered density was measured by an Archimedes method. The degree of oxidation $(\xi)$ was calculated by the equation, $1-\left(I / I^{\circ}\right)$, where $I$ and $I^{\circ}$ were the integrated X-ray diffraction intensity of the 
(110) reflection of NiAl for the oxidized and sintered materials, respectively. The three-point bending test was conducted at the span of $10 \mathrm{~mm}$ and the cross-head speed of $0.5 \mathrm{~mm} \cdot \mathrm{min}^{-1}$. The morphology of oxidized surface and fracture surface were observed by scanning electron microscopy and color laser microscopy (KEYENCE, VE9800). The fracture toughness values were determined by indentation fracture (IF) and indentation strength (IS, load: 98N) methods. The IF values were calculated by Evans' equation using the crack length determined in the SEM images at the magnification $\times$ 100. The IS values should be agreed with that of the as-sintered materials when the median crack runs across the layer interface.

\section{3 .RESULTS AND DISCUSSION}

3.1 Oxidation and development of metamorphic layer

The NA01 composition was densified to $99.2 \%$ at $1430^{\circ} \mathrm{C}$. The microstructure of the sintered material is shown in Fig.1(a). The bright grains in the photo were the $\mathrm{NiAl}$ grains with the size of $0.2-2 \mu \mathrm{m}$. The NiAl grains were dispersed as the assemblage with the size of $10 \sim 20 \mu \mathrm{m}$ derived from NA10 composition inside the dashed line in Fig.1 (a). The size of matrix $\mathrm{Al}_{2} \mathrm{O}_{3}$ was 2-4 $\mu \mathrm{m}$ and the size of $\mathrm{Al}_{2} \mathrm{O}_{3}$ grains inside the assemblage was about $1 \mu \mathrm{m}$ in the higher magnification images. The dispersant NA10 grains were oxidized at $1250^{\circ} \mathrm{C}$, and provided the "Escher-like" structure and the network of the oxidation product, $\mathrm{NiAl}_{2} \mathrm{O}_{4}$, along the boundary of the matrix $-\mathrm{Al}_{2} \mathrm{O}_{3}$ on the surface. The oxidation of NiAl represented by the equation (1) and (2) requires the additional $\mathrm{Al}_{2} \mathrm{O}_{3}$ to react with $\mathrm{NiO}$. This provides the driving force for the boundary diffusion of $\mathrm{NiO}$ to form $\mathrm{NiAl}_{2} \mathrm{O}_{4}$ at the matrix-boundary, resulting in the network. However, the network was not completely connected to each other at the oxidation time 2.5 and $3 \mathrm{~h}$, arrows in Fig.1 (b) and (c).

Prolonged oxidation promoted the development of the network structure, and the well-established network had been obtained at 5h-oxidation (d). Further oxidation resulted in the shrinkage of the "Escher" and the disappearance of the network at $6.5 \mathrm{~h}$ (e) and converted to the film-structure at $20 \mathrm{~h}$ (f). This is probably caused by the diffusion of the oxidation product from the surface to the inner bulk. When a certain amount of the oxidation product is accumulated on the surface, the increased chemical potential should cause the diffusion toward the inner parts of the material. The unique crack propagation behavior different from the original NA01 composite is supposed in the metamorphic layers consisting of $\mathrm{Al}_{2} \mathrm{O}_{3}$ grains and the grain boundary substituted by the oxidation product $\mathrm{NiAl}_{2} \mathrm{O}_{4}$.

The oxidation product $\mathrm{NiAl}_{2} \mathrm{O}_{4}$ showed the characteristic blue-green color. Then, the fracture surface was observed by a color LASER microscope. Figure 2 shows the blue-color enhanced image of the materials oxidized for $5 \mathrm{~h}$ and $6.5 \mathrm{~h}$. The oxidation was limited on the thin metamorphic layer within $10 \mu \mathrm{m}$ for the 5 h-oxidized material but expanded to $31 \mu \mathrm{m}$ for the 6.5h-oxidized one.

The degree of the oxidation ( $\xi$ ) obtained by XRD intensity and the thickness of the metamorphic layer $(\delta)$ obtained from the color LASER images are plotted against the oxidation time in Fig.3. The degree of oxidation was $72 \%$ with the thin metamorphic layer for $5 \mathrm{~h}$, but increased to $94 \%$ abruptly at $6.5 \mathrm{~h}$. The thickness $\delta$ also increased at $6.5 \mathrm{~h}$-oxidation, where the surface NiAl was almost oxidized. The diffusion of the oxidation product into the bulk should result in the decrease in the thickness of $\mathrm{NiAl}_{2} \mathrm{O}_{4}$ boundary in compensation to the increase in the thickness of the metamorphic layer.

The oxidation reactions (1) and (2) result in the volume expansion due to the difference in density of NiAl $\left(\rho_{\mathrm{NA}}=5.89 \mathrm{~g} \cdot \mathrm{cm}^{-3}\right)$ and $\mathrm{NiAl}_{2} \mathrm{O}_{4} \quad\left(\rho_{\mathrm{sp}}=4.51 \mathrm{~g} \cdot \mathrm{cm}^{-3}\right)$ according to equation(3).
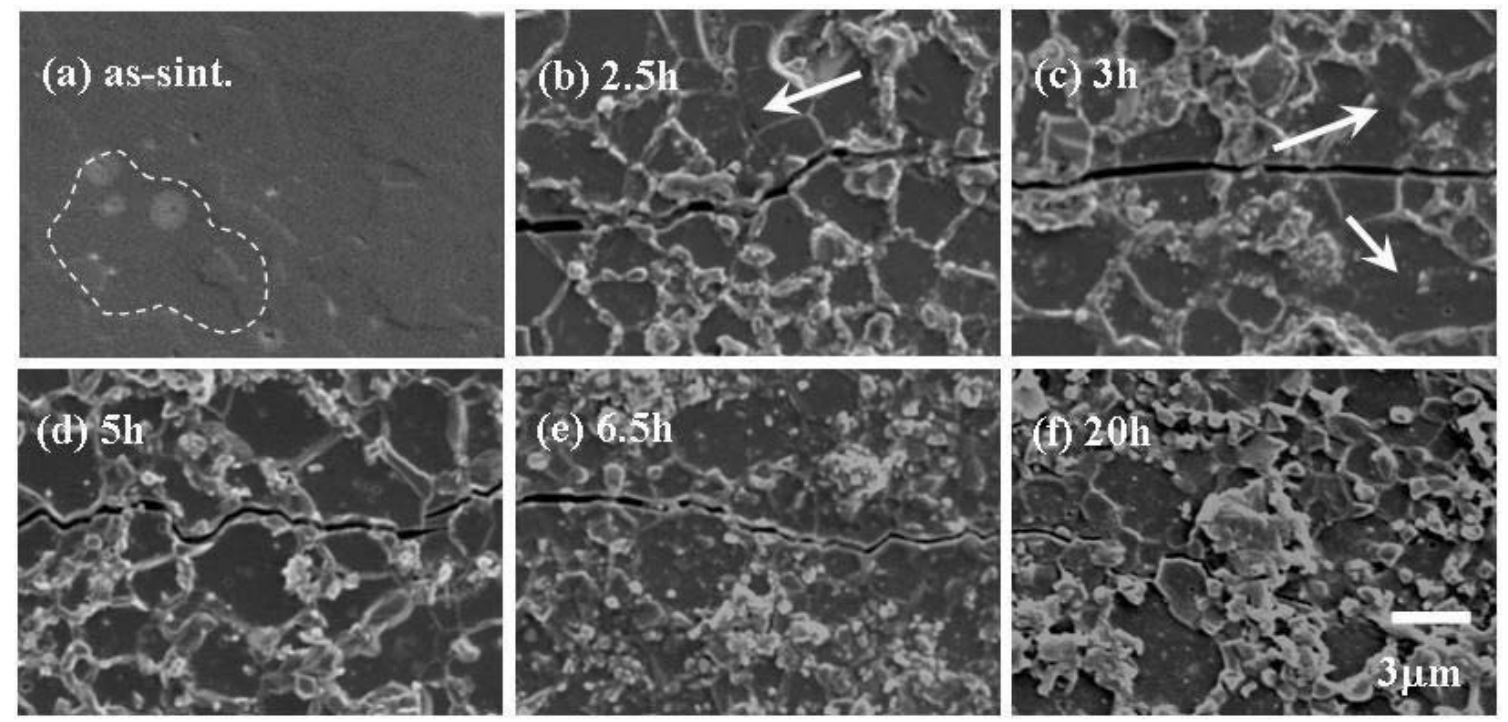

Fig.1 Surface morphology of sintered and oxidized materials with the crack propagation from the Vickers indents. 

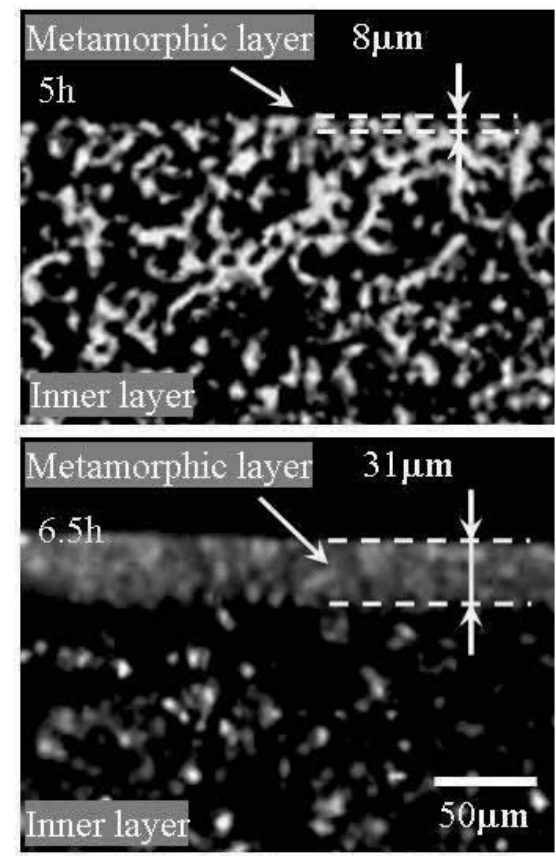

Fig.2 Fracture surface with the enhancement of the metamorphic layer.

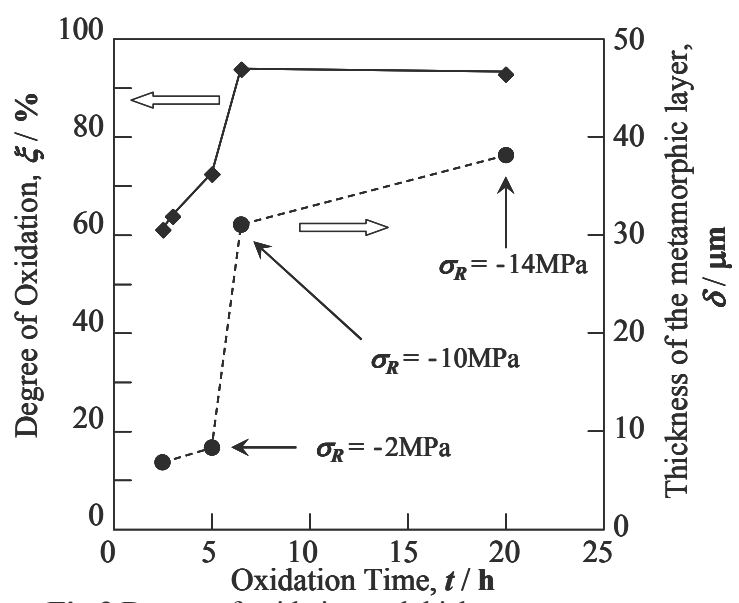

Fig.3 Degree of oxidation and thickness of the metamorphic layer.

where $X$ is a volume fraction of NiAl. $\rho$ and $M$ is the density and formula weight, respectively.

The subscript of $\mathrm{A} 0, \mathrm{NA}$ and SP mean $\mathrm{Al}_{2} \mathrm{O}_{3}, \mathrm{NiAl}$ and $\mathrm{NiAl}_{2} \mathrm{O}_{4}$.

This volume expansion can be converted to the compressive stress $\left(\sigma_{\mathrm{R}}{ }^{\mathrm{cal}}\right)$ in the metamorphic layers as a function of $\delta$ and $\xi$ in the following equation (4) [2].

$\sigma_{\mathrm{R}}{ }^{\mathrm{cal}}=\left(\Delta V^{1 / 3}-1\right) \cdot E \cdot \delta[(1-v) d]^{-1}$

where $\delta$ and $\xi$ are the thickness of the metamorphic layer and specimen. The elastic modulus $(E=398 \mathrm{GPa})$ and Poisson ratio $(v=0.237)$ were determined by a ultrasonic pulse method. For this work expecting the low compressive stress, the $\sigma_{\mathrm{R}}{ }^{\text {cal }}$ was calculated as $-2 \mathrm{MPa}$ for $5 \mathrm{~h}$-oxidized material and $-14 \mathrm{MPa}$ even for $20 \mathrm{~h}$-oxidized one.

\subsection{Mechanical properties and crack propagation}

The effective fracture toughness $\left(K_{\mathrm{c}-\mathrm{eff}}\right)$ determined by the IF method for the as-sintered and oxidized materials is shown in Fig.4 with the estimated values using equation (5).

$K_{\mathrm{c}-\mathrm{eff}}{ }^{\mathrm{cal}}=K_{\mathrm{IC}}{ }^{\mathrm{o}}-2 \sigma_{\mathrm{R}}{ }^{\mathrm{cal}}\left(c^{*} / \pi\right)^{1 / 2}$

where $K_{\mathrm{IC}}{ }^{0}\left(3.5_{6} \mathrm{MPa} \cdot \mathrm{m}^{1 / 2}\right)$ is the fracture toughness of the as-sintered material and $c^{*}$ is the crack length of the oxidized ones.

The obtained $K_{\text {c-eff }}$ values were evidently higher than the estimation, meaning the toughening mechanism irrespective of compressive stress. The $K_{\text {c-eff }}$ values increased in the progress in oxidation within the oxidation time of $5 \mathrm{~h}$, where the oxidation was limited in the thin surface layer, and then decreased with the further oxidation showing the diffusion of $\mathrm{NiAl}_{2} \mathrm{O}_{4}$ into the bulk. The highest $K_{\text {c-eff }}$ of $6.4 \mathrm{MPa} \cdot \mathrm{m}^{1 / 2}$ was obtained for the 5 h-oxidized material. However, the $K_{\text {c-eff }}$ determined by the IS method was smaller than the IF value and agree with $K_{\text {ceff }}^{\text {cal }}$ comparable of $K_{\mathrm{IC}}{ }^{\circ}$.

In the IS method, the depth of induced crack was $180 \mu \mathrm{m}$, which was deeper than the metamorphic layer obviously. The values of fracture toughness by IS method agreed with the value of sintered composite. Then, the improvement of the fracture toughness was caused by the development of metamorphic layer, especially by the well-developed network of $\mathrm{NiAl}_{2} \mathrm{O}_{4}$.

Fracture surface of the sintered and oxidized materials are shown in Fig.5. Fracture surface of sintered materials showed the both transgranular and intergranular crack propagations. Fracture surface of $2.5 \mathrm{~h}$ - and 3 h-oxidized materials with thin metamorphic layer showed the similar propagation, but the fraction of intergranular propagation increased at $3 \mathrm{~h}$. Obvious intergranular fracture was observed for 5h-oxidized material with the well-developed network of thick $\mathrm{NiAl}_{2} \mathrm{O}_{4}$. This feature was maintained at $6.5 \mathrm{~h}$ partly, but disappeared at $20 \mathrm{~h}$. This disappearance of the intergranular fracture was corresponded to the shrinkage of the network structure observed in Fig.1 (e) and (f). The evident intergranular fracture to improve the

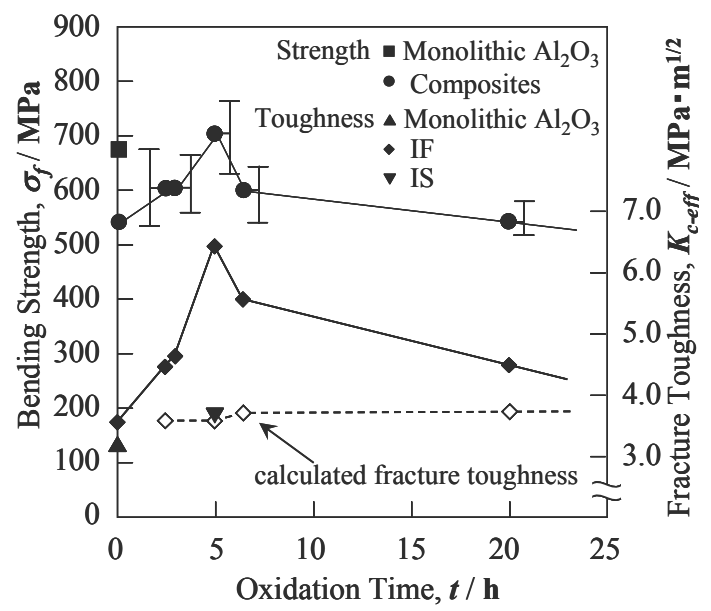

Fig.4 Bending strength and fracture toughness as functions of oxidation time. 

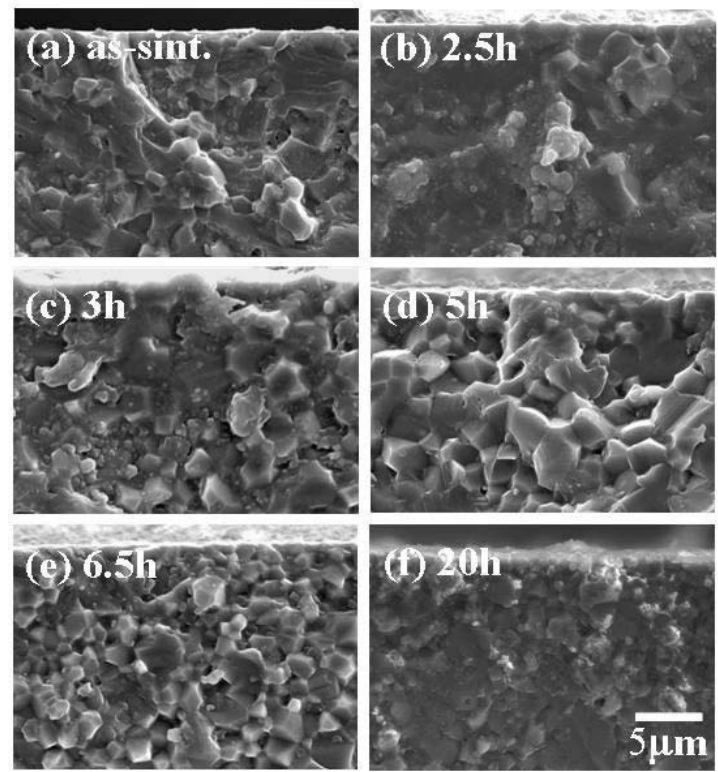

Fig. 5 Fracture surface of sintered and oxidized materials.

fracture toughness was strongly related to the obvious deflection of the cracks in Fig.1(c). It is considered that the soft and weak $\mathrm{NiAl}_{2} \mathrm{O}_{4}$ (Vickers hardness, $\mathrm{Hv}$ $=14.8 \mathrm{GPa}$ against $16.8 \mathrm{GPa}$ for $\mathrm{Al}_{2} \mathrm{O}_{3}$ and $\sigma_{\mathrm{f}}=288 \mathrm{MPa}$ against $672 \mathrm{MPa}$ for $\mathrm{Al}_{2} \mathrm{O}_{3}$ ) substituting the grain boundary in the metamorphic layers introduces the cracks to the boundary network to cause the effective crack deflection improving the fracture toughness. The compressive stress of -10 and $-14 \mathrm{MPa}$ for $6.5 \mathrm{~h}$ - and 20h-oxidized materials compensated the reduction of the deflection effect to prevent the drastic degradation of the fracture toughness.

The fracture strength is also shown in Fig.4. The strength of sintered material, 539MPa was lower than $672 \mathrm{MPa}$ for the monolithic $\mathrm{Al}_{2} \mathrm{O}_{3}$ prepared from the matrix powder. This was caused by the lower density of $99.2 \%$ and the relatively large size of NA10 assembles. However, the strength was improved to $601 \mathrm{MPa}$ by the oxidation for $2.5 \mathrm{~h}$, and attained to the maximum value of $701 \mathrm{MPa}$ for $5 \mathrm{~h}$-oxidized material. The dependence of $\sigma_{\mathrm{f}}$ on the oxidation time was similar to that of $K_{\text {c-eff. }}$ It was considered that the increased resistance to crack propagation due to the increased deflection ability and/or the compressive stress, it was insufficient, improved the strength.

\section{CONCLUSIONS}

The oxidation-derived microstructural development and the mechanical properties of $\mathrm{Al}_{2} \mathrm{O}_{3}$-based material dispersing a very small amount (1vol\%) of NiAl has been studied. The development of the network structure substituting the boundary of the matrix $-\mathrm{Al}_{2} \mathrm{O}_{3}$ with the oxidation product $\mathrm{NiAl}_{2} \mathrm{O}_{4}$ induces the enhanced crack deflection to improve the fracture toughness and strength from $3.6 \mathrm{MPa} \cdot \mathrm{m}^{1 / 2}$ and 539MPa (as-sintered) to $6.4 \mathrm{MPa}$. $\mathrm{m}^{1 / 2}$ and $701 \mathrm{MPa}$ at the oxidation time of $5 \mathrm{~h}$. When the oxidation of the surface $\mathrm{NiAl}$ is completed, the diffusion of $\mathrm{NiAl}_{2} \mathrm{O}_{4}$ into the inner bulk results in the shrinkage of network and the decrease in the thickness of the boundary $\mathrm{NiAl}_{2} \mathrm{O}_{4}$. The deflection effect fades away for the structure. However, the compressive stress due to the volume expansion of the metamorphic layer prevents the drastic degradation.

\section{ACKNOWLEDGEMENTS}

This work has been conducted with the financial assistance of the Grant-in-Aid (No.2036410) of the ministry of Education, Culture, Sports, Science and Technology, Japan. The authors also wish to sincere thanks to the kind assistance.

\section{REFERENCES}

[1] O. Abe, J. Yamada, "Preparation of Multilayer Composites by Combination of Centrifugal Filter Pressing and Rate-Controlled Sintering", J. Ceram. Soc. Japan, 102, 627-632 (1994).

[2] R. Sathyamoorthy, A. V. Virkar, and R. A. Culter, "Damage-Resistant SiC-AIN Layered Composites with Surface Compressive Stress“, J. Am. Ceram. Soc.,75, 1136-1141 (1992).

[3] O. Abe, Y. Ohwa, "Oxidation of $\mathrm{NiAl} / \mathrm{Al}_{2} \mathrm{O}_{3}$ composites for controlled development of surface layers and toughening" Solid State Ionics, 172, 553-556 (2004).

[4] O. Abe, S. Takata, and Y. Ohwa, "Toughening of NiAl-alumina composites by self-constructed compressive surface layers under Oxidation" J. Eur. Ceram. Soc., 24, 489-494 (2004).

[5] O. Abe, Y. Ohwa, and Y. Kuranobu, "Possibility of enhanced strength and self-recovery of surface damages of ceramics composites under oxidative conditions", J. Eur. Ceram. Soc., 26, 689-695 (2005).

[6] D. B. Marshall, J. J. Ratto, and F. F. Lange, "Enhanced Fracture Toughness in Layered Microcomposites of $\mathrm{Ce}-\mathrm{ZrO}_{2}$ and $\mathrm{Al}_{2} \mathrm{O}_{3}{ }^{*}, \mathrm{~J} . \mathrm{Am}$. Ceram. Soc., 74 [12], 2979-2987 (1991).

(Received December 12, 2008 ; Accepted January 31, 2009 